\title{
Research on the Application of Cloud Computing in the Future Market in China
}

\author{
Linzheng Ruan ${ }^{1, *}$ \\ ${ }^{1}$ HangZhou XueJun highSchool Zijingang campus, Hangzhou Zhejiang China, 310000 \\ *Corresponding author. Email:1106561003@qq.com
}

\begin{abstract}
In recent years, cloud computing is becoming more and more important. So, this thesis is about analyzing the future market of cloud computing in China in the future. And the main Research object is the market of cloud computing in China. All the data are acquired online. And after the analysis, cloud computing will show a very potential trend in the future. And the future of the market is bright.
\end{abstract}

Keywords: Cloud computing, market, the application of cloud computing, market size

\section{INTRODUCTION}

In contemporary society, cloud computing is becoming more and more significant for its properties. Through cloud computing, tons of data can be processed in a short period of time to achieve powerful network services. The value of cloud computing lies in its high flexibility, scalability, and high-performance ratio. So, more and more attention is paid to this technology. Many laws and legislations appear at the same time. In this article, some situations and environments of cloud computing will be analyzed and the future markets in China for the cloud computing will be figured out in order to invest this field in a more efficient way.

\section{THE CURRENT STATUE OF THE CLOUD COMPUTING}

Commonly, its service types are divided into three groups, namely Infrastructure as a Service (IaaS), Platform as a Service (PaaS) and Software as a Service (SaaS). These three cloud computing services are sometimes called cloud computing stacks because they build stacks, and they are on top of each other. The following is a brief introduction of them
First, Infrastructure as a Service (IaaS)

Infrastructure as a service is one main service category. It provides virtualized computing resources including virtual machines, storage, networks, and operating systems to individuals or organizations of cloud computing providers.

Second, Platform as a Service (PaaS)

Platform as a service is a service category that gives developers a platform for building applications and services through the global Internet. Paas provides an ondemand development environment for developing, testing and managing software applications.

Third, Software as a service (SaaS)

Software as a service is also a category of its services, providing on-demand software payment applications via the Internet, cloud computing providers hosting and managing software applications, and allowing its users to connect to the application and access the application through the global Internet [1]

And according to its deployment model it can be divided into private cloud, community cloud public cloud and hybrid cloud. 


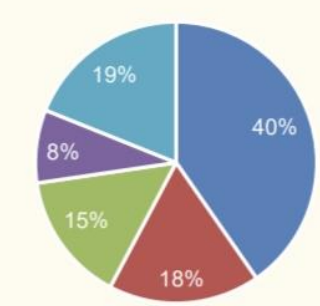

-Alibaba Cloud " Huawei Cloud " Tencent Cloud "Baidu Al Cloud = Others

Figure 1China cloud computing market share in 2020 quarter 4 [1]

In 2020 Q4, Alibaba held the biggest proportion of the cloud computing market $(40 \%)$. And many different companies held some different proportion of the market.

There are also some less important corporations such as AWS Microsoft and so on. They are including in the other proportion of the market.

The scale of Chinese computing market only accounts for $1 / 6$ in the world, with a strong late-comer advantage and large room for development. In 2018, the scale of China's cloud computing market reached 90.7 billion yuan, a year-on-year growth rate of $31 \%$. It is expected that the market size will exceed 100 billion in 2019. [2]

The evolution of the industry.

In the U.S, the concept was introduced in 2005 based on the virtual technology and some other basic technology. Some internet technology companies started to conduct some experiments about developing the prototype of the cloud computing. During 2005 and 2008, the technology was spread among the enterprise. After that, the widely application of cloud computing accelerated the growth of the spread. Since 2011, this technology has become prevalent in the country. Some infrastructures were transferred into the public cloud. So the quick development of cloud computing helps with the demand for consumers and capital support for enterprises.

In China, the development of cloud computing is not rapid as it is in American. The enterprise has tried to learn the technology since 2007. However, the cloud computing did not commercialize successfully. And two years later, with the help of policy and investment from? Some local government started to develop some cloud database centre. From 2013 to 2015, some multinational corporation servers started the business of cloud computing in China. Local enterprises began to learn the cloud system and many corporations show great potential. The wide use of cloud computing has been started since 2015 for its conveniences and diverse functions.

\section{MAIN PROBLEMS OF THE MARKET}

As the cloud computing is becoming more and more prevalent and popular among consumers and enterprises, there are still several problems for the cloud computing.

\subsection{Data security and privacy}

In the cloud computing system, the user's data is stored in the cloud. How to ensure that the user's data is not illegally accessed or leaked by wireless interconnection technology and network is two important issues that the system must solve, that is, data security and privacy issues. If it is to prevent data loss, data backup is generally adopted, that is, to write at least two copies of the data; if it is to prevent misoperation, it can be controlled by data log information and delayed replication; if it is to prevent hackers, it is often the public cloud needs to be encrypted. These precautions can only control some of the problems we can see, and there are some security problems that we cannot predict threaten the development of cloud computing. Therefore, the data security and privacy protection of cloud computing is a problem that must be considered and overcome in the development of cloud computing platforms.

\subsection{Missing standards}

With the rapid development of domestic cloud computing technology, the need for the construction of national cloud computing standards have become more urgent. If there is no standard, the development of the cloud computing industry will be difficult to achieve norms and health, and it will be difficult to achieve scale and industrialization.

\subsection{Cloud governance}

With the development of cloud computing technology, it has also brought about increasingly severe effective management risks. The increasing number and variety of cloud data has given birth to "cloud governance." Cloud governance issues focus on two aspects, one is the governance of the service itself, and the other is the system governance behind the service. 
The significance of cloud governance itself is not to have or display a lot of data, but to make society run more smoothly, social services more effective, and cloud computing to better serve social development.

\section{THE ACCESSIBILITY OF THE CLOUD COMPUTING}

Now, many areas apply the cloud computing. At present, some domestic IT companies are also trying to explore cloud applications in finance. For example, Digital China seizes the opportunity of the China Banking Regulatory Commission to encourage the opening of rural financial institutions, builds a rural financial information platform, and integrates functional modules shared by rural financial institutions to provide rural financial services. Institutions provide rental services and charge on-demand; domestic call platform integrators such as Infobird and Qingniu provide insurance companies with call platform cloud services through Internet-based call software, so that small and medium insurance companies' call centres can use expensive self-built platforms Shift to a convenient way of leasing, so as to realize the commercial value of reducing costs and improving service quality. Additionally, this technology is also widely used in cloud desktop. Through any other device connected to the network, it can access cross-platform applications.

In the future, more and more fields can apply this kind of technology. For instance, it can be applied more ingame cloud. Since the game has the properties of fast development speed, high developing cost and high risk, it will face a lot of problems in the closed beta and public beta. So, the cloud computing can be applied since it is of high efficiency while the cost of it is low. It is also easy to expand its field since it is scalable. Also, many other areas use this kind of technology as well, like medical, education, foreign trade, governance and so on, since this technology is virtual, flexible liable and etc.

\subsection{The macroeconomic situation of cloud computing}

In China, there are many advantages to the development of cloud computing.

\subsubsection{The help of governments and cities accelerate the develop of cloud computing}

According to a report in China, some leading cities in the IT industry such as Beijing, Shanghai, Shenzhen, Fujian, Jiangsu, Zhejiang, etc. have successively launched cloud computing industry development plans, built infrastructure, and then a number of cities such as Wuxi, Nanjing, Hangzhou, Harbin actively cooperate with leading companies to expand application.[3] so with the help of government and some universities, the stress of finance and technology of developers might be released, the future development of cloud computing will show great potential.

\subsubsection{Some support of policy and laws.}

More and more attention is paid on this field since the cloud computing is powerful and provide strong support for consumer and enterprise. So, many policies and laws are set up to help with the develop of cloud computing. For instance, At the Fifth Plenary Session of the Seventeenth Central Committee, the State Council issued the Decision of the State Council on Accelerating the Cultivation and Development of Strategic Emerging Industries, proposing to vigorously develop strategic emerging industries, and the proportion of GDP will rise from $8 \%$ in 2015 to $15 \%$ in 2020 . As an important branch of strategic emerging industries, the new generation of information technology has also received strong support from the "Decision", which mentions the need to promote the research and development and demonstration applications of the Internet of Things and cloud computing[ 4] . Therefore, the policy will provide the development of cloud computing with strong help.

\subsubsection{Development of Technology.}

Since the cloud computing is showing its significance in the recent years, more and more cooperation is made among the university, research institutions and enterprise, and many experiments are conducted. According to a report, based on the distributed file system, Tsinghua University has designed and developed cloud storage services for data storage and sharing for campus network users, and has achieved results in building storage clouds. The University of Science and Technology of China has designed a data integrity verification protocol to protect user privacy in terms of remote data integrity and authentication technology in the cloud computing environment; it has proposed a more rigorous authentication scheme for the shortcomings and security vulnerabilities of existing remote identity authentication schemes. [3] Much other research is conducting, and this kind of cooperation and research will accelerate the development of the cloud computing of our country efficiently.

\subsubsection{The economic situation.}

Since the economics of China is developing at a very fast speed, the GDP of China is growing at a very fast speed. In 2020, gross domestic products of China is 101599 billion yuan(National data). And the growth rate of it shows great result. The growth rate is approximately remaining at $6 \%$ (national data) except for 2020(covid19). And the expenditure for new product development in high-tech industries is also increasing, from 355893 million yuan in 2016 to 540748 million in 2019 . (National data).[5] Additionally, the market for cloud 
computing are still very wide for the enterprise. in China, the study and research about cloud computing is five years later than in American. So, the Market size is not so large as those in American and the enterprise still have efficient area to discover and cultivate.

\subsubsection{Future trend of China cloud computing.}

The trend of market is clear. Since the cloud computing has a very wide market, it is supposed that the market size and the growth rate will both grow at a very rapid speed. As for the demand, more and more consumers will choose to apply cloud computing. This technology has been widely applied in many fields, likes finance, education, medical and so on. For educations, the cloud computing can bring the students more and more methods to accomplish their courses likes having the online curriculums. For teachers they can make the standard through cloud computing, and they will satisfy some demands of students more conveniently and efficiently. So, the demand will show great potential. For the supply, as the enterprise is now having many advanced technologies and blueprints, the income of cloud computing is now growing from loss. So, the providers may try to invest more in this field and the supply may also grow. And since many cloud computing providers sell their products as a subscription, the demand will keep growing in the long term and will bring providers with long term consumers.

\subsection{Challenges and risks}

Enterprise cloud migration is not as expected

The deepening of the mobile Internet, the large-scale replication of the Internet of Things, and the digital transformation of traditional enterprises are the three major factors driving the development of the cloud computing industry. If companies fail to go to the cloud as expected, and the demand for cloud computing does not meet expectations of the producer, this will result in a slowdown in the revenue and profit growth of industry chain companies.

The capital expenditure of cloud giants is less than expected

Cloud giants are in the middle of the industry chain, with deep barriers and strong bargaining power. The capital expenditure of cloud giants drives the upstream and downstream development of the industry chain. If the growth and intensity of capital expenditure falls short of expectations, it will result in lower-than-expected revenue and profit in upstream industries.

Sino-US trade frictions Sino-US trade frictions may expose some downstream customers or partners to U.S. trade sanctions and export control, thereby affecting the company's operating results. According to an experiment, the trade fraction will lead to a decrease of $0.93 \%$ and $1.73 \%$ in Chinese export and import department. Also, in American the impact of trade friction is great. The impact is $-0.96 \%$ and $-0.87 \%$ in the export and import department. [6], so the trade frictions may bring some adverse impact on the cloud computing.

\section{CONCLUSION}

The future market for cloud computing is bright. There is still a lot of potential for this field, and this will lead to a growth in the market size, growth rate and the revenue for the providers. As the demand is growing, more and more people may choose to apply this technology. The supply-sides of cloud computing are now more and more mature. And in recent years, its market size may get close to the size of American cloud computing. The number of population and enterprises in China is extremely huge and with the economic development of China, this tendency is predictable.

\section{REFERENCES}

[1] Wang Xiong.The history and advantages of cloud computing $[\mathrm{J}]$.Computer

Networks, 2019,45(2):44.

[2] Zhiyan Consulting "2019-2025 China Cloud Computing Industry Market Analysis Forecast and Investment Direction Research Report".

[3] Gong Qiang my country Cloud Computing Development Research Summary 1, 2 (1. Institute of Engineering Mechanics, China Earthquake Administration, Harbin 150080; 2. Heilongjiang Provincial Non-ferrous Metal Geological Exploration Bureau, Harbin 150090) 2-3.

[4] State Council "Decision of the State Council on Strengthening the Cultivation and Development of Strategic Emerging Industries"national data.

[5] Zhou Zhengning, Shi Xinlu International Economic and Trade Exploration vol.35 mo.2 feb.2019 The impact of trade frictions on China and the United States: An analysis based on the dynamic GTAP model 27-28. 